\title{
$\alpha 4 \beta 7$ Integrin (LPAM-1) is Upregulated at Atherosclerotic Lesions and is Involved in Atherosclerosis Progression
}

\author{
Kangkang Zhi ${ }^{a}$ Mengfan Lib Xiaoping Zhang ${ }^{c, d}$ Zhiwei Gao Jun Bai ${ }^{a}$ \\ Yongfa Wu Sili Zhou Maoquan Lic,d Lefeng $\mathrm{Qu}^{\mathrm{a}}$ \\ ${ }^{a}$ Department of Vascular and Endovascular Surgery, Changzheng Hospital, ${ }^{b}$ Department of Vascular \\ Surgery, Putuo District Central Hospital, cDepartment of Interventional Radiology, Tenth People's \\ Hospital of Tongji University, IInstitute of Medical Intervention Engineering, Tongji University, Shanghai, \\ China
}

\author{
Key Words \\ $\alpha 4 \beta 7$ integrin $•$ ApoE $^{-/-}$mice $\cdot$ Atherosclerosis $•$ Phagocytosis
}

\begin{abstract}
Background/Aims: Integrin activation and lymphocyte migration to the vascular intima is a key event in early atherosclerosis. $\alpha 4 \beta 7$ integrin (LPAM-1) and its ligand, mucosal addressin cell adhesion molecule (MAdCAM-1) are known to play an important role in homing of activated lymphocytes to gut-associated lymphoid tissues. However, it is unclear whether $\alpha 4 \beta 7$ integrin is involved in the pathogenesis of atherosclerosis. Methods: The expressions of $\alpha 4 \beta 7$ integrin and its ligands in atherosclerosis plaques from 12 week high fat diet (HFD) fed $\mathrm{ApoE}^{-/-}$and C57BL/6 mice were examined using immunofluorescent and immunohistochemical assays, respectively. We also generated $A p o E / \beta 7$ double deficient mice and compared atherosclerotic lesion development in $\beta 7^{+/+} \mathrm{Apo} \mathrm{E}^{-/-}$and $\beta 7^{-/-} \mathrm{ApoE^{-/- }}$ mice that were fed with HFD for 12 weeks. Results: We found an upregulation of $\alpha 4 \beta 7$ integrin and its ligands VCAM- 1 and MAdCAM-1 at atherosclerosis plaques in Apolipoprotein $E$ deficient $\left(A p o E^{-/-}\right.$) mice fed with HFD for 12 weeks. Over the 12 week HFD period, peripheral blood lymphocyte (PBL) expression of $\alpha 4 \beta 7$ integrin increased in parallel with aortic lesion size. A removal of $\alpha 4 \beta 7$ integrin by genetic deletion of the $\beta 7$ chain in the $\mathrm{ApoE}^{-/-}$mouse resulted in a markedly decreased 12 weekHFD atherosclerotic plaque area. $\beta 7^{-1-} \mathrm{ApoE}^{-/-}$macrophages showed reduced acetylated and native LDL uptake and phagocytic activity, revealing possible roles for $\alpha 4 \beta 7$ at two distinct stages of macrophage dysfunction during atherogenesis. Finally, a reduced activity of integrin downstream signalling components focal adhesion kinase (FAK) and MAPK/ERK1/2 in macrophage indicates their possible engagement during $\alpha 4 \beta 7$ integrin signalling in atherosclerosis. Conclusions: Together our results reveal a critical role of $\alpha 4 \beta 7$ in diet-induced atherosclerosis in mouse.
\end{abstract}

Kangkang Zhi and Mengfan Li contributed equally to this work. 


\section{Cellular Physiology and Biochemistry}

Cell Physiol Biochem 2014;33:1876-1887

DOI: 10.1159/000362965

Zhi et al: $\alpha 4 \beta 7$ Integrin Involved in Atherosclerosis

\section{Introduction}

Extravasation of circulating lymphocytes into lymphoid and extra-lymphoid tissue is essential in normal immune surveillance and inflammation, but also contributes to chronic inflammatory diseases such as atherosclerosis. Atherosclerosis is initiated by apolipoprotein B-containing lipoprotein (apoB-LP) accumulation in the matrix beneath the arterial endothelial cell layer, which triggers an early inflammatory response in the overlying endothelial cells. Activated endothelial cells secrete chemokines that interact with cognate receptors on lymphocytes and promote directional migration in a highly coordinated 'multistep adhesion cascade' involving cellular adhesion molecules (CAMs) differentially expressed on lymphocytes and endothelial cells [1]. The majority of monocytes in early atherosclerotic lesions differentiate into cells with macrophage-and/or dendritic cell-like features [2, 3], which soon become lipid-loaded after ingesting and processing apoB-LPs, resulting in foam cell formation. The lesion progresses into a dangerous plaque as chronic inflammation drives further leukocyte recruitment and macrophage foam cells undergo apoptosis but are not effectively cleared by phagocytosis, leading to necrosis. When the lesion ruptures, thrombogenic material becomes exposed and causes platelet aggregation and thrombus formation, ultimately manifesting as acute coronary syndrome, myocardial infarction or stroke [4].

Several lines of evidence support a crucial role of CAMs in the development of atherosclerosis and plaque instability. Not only has the expression of L-selectin, $\alpha v \beta 3$ integrin and integrin ligands vascular cell adhesion molecule-1 (VCAM-1) and intercellular adhesion molecule-1 (ICAM-1) been consistently observed in atherosclerotic plaques [5-8], but studies in gene-targeted mice have revealed pro- as well as anti-atherogenic roles for CAMs, including a number of $\beta 1-3$ integrin family members and their ligands [4, 9-12]. Integrins are cell-surface heterodimers that mediate interactions between the extracellular environment and platelets, inflammatory cells, and the vasculature. $\beta 1, \beta 2$ and $\beta 3$ integrins are known to be relevant to distinct atherogenic stages. In white blood cells, $\alpha \mathrm{L} \beta 2$ in leukocytes and $\alpha \mathrm{X} \beta 2$ in monocytes/macrophages interact with endothelial expressed ICAM upon activation by selectins [13-15]. At a subsequent stage, $\alpha 4 \beta 1$ integrin interaction with endothelial VCAM drives lymphocyte cell shape changes and migration at the surface of the endothelium to reach a junction [16]. Several $\beta 1$ integrin members are also expressed on smooth muscle cells [17]. Platelets express integrins from the $\beta 3$ family, $\alpha 2 b \beta 3$ being specific for platelets and involved in fibrin formation and $\alpha \mathrm{V} \beta 3$ being more widely expressed and functioning in cell survival, migration and proliferation [18].

While the involvement of the $\beta 1, \beta 2$ and $\beta 3$ integrin families in atherosclerosis has been widely studied, that of the $\beta 7$ integrin family, comprised of $\alpha 4 \beta 7$ (also known as lymphocyte Peyer's patch adhesion molecule, LPAM-1) and $\alpha \mathrm{E} \beta 7$ (also known as the human mucosal lymphocyte antigen HML-1) is unknown. $\alpha 4 \beta 7$ integrin and its ligand MAdCAM- 1 play an important role in homing activated lymphocytes to gut-associated lymphoid tissues. $\alpha 4 \beta 7$ integrin is expressed on several cells, such as lymphocytes, macrophages, and eosinophils, and its ligands include mucosal addressin cell adhesion molecule-1 (MAdCAM-1) and VCAM1 [19-21]. MAdCAM- 1 is the main ligand of $\alpha 4 \beta 7$ integrin and is expressed on Peyer's patches of intestines, high endothelium venules of mesenteric lymph nodes, and vascular endothelial cells of gut lamina propria [22-24]. However, it is not known whether $\alpha 4 \beta 7$ integrin and its main ligand MAdCAM-1 are expressed during atherogenesis or relevant to atherosclerosis.

The notion of $\alpha 4 \beta 7$ involvement in atherogenesis finds support in a study showing that murine endothelial cells can be induced to express high levels of MAdCAM-1 in response to pro-inflammatory cytokines [25]. Given that $\alpha 4 \beta 7$ is the exclusive ligand of MAdCAM-1, we sought to determine whether $\alpha 4 \beta 7$ integrin contributes to atherosclerosis. We found that $\alpha 4 \beta 7$ and its ligands MAdCAM- 1 and VCAM- 1 are upregulated in diet-induced atherosclerotic lesions in the $\mathrm{ApoE}^{-/}$mouse model of atherosclerosis. Upon detecting a substantial upregulation of $\alpha 4 \beta 7$ and its ligands in atherosclerotic plaques we investigated its functional relevance in $\beta 7 \% \mathrm{ppoE}^{-/}$double deficient mice. We found that upon removal of $\beta 7$, 


\section{Cellular Physiology and Biochemistry}

Cell Physiol Biochem 2014;33:1876-1887

DOI: $10.1159 / 000362965$

Publisnea onime: June 23,2014

(C) 2014 S. Karger AG, Basel

www.karger.com/cpb

ApoE $/$ mice on a 12 wk HFD had markedly decreased vascular disease. Our subsequent in vitro studies suggest that $\alpha 4 \beta 7$ integrin may play roles in macrophage lipid uptake as well as phagocytosis, possibly by activating signal transduction molecules FAK and ERK1/2.

\section{Materials and Methods}

\section{Ethics Statement}

This work was carried out in strict accordance with international ethical guidelines and the National Institutes of Health Guide concerning the Care and Use of Laboratory Animals. The experiments were carried out with the approval of the Animal Experimentation Ethics Committee of the Second Military Medical University.

Animals

ApoE $/$ and $37 \%$ [26] mice on a C57BL/6 background were purchased from Jackson Laboratory (Bar Harbor, ME, USA) and C57BL/6 mice were obtained from SLAC Laboratory Animal Co. Ltd (Shanghai, China). Mice were housed and manipulated according to the protocols approved by the Laboratory Animal Center of the Second Military Medical University. To generate ApoE/ $\beta 7$ double deficient mice, ApoE ${ }^{-/}$and $\beta 7^{-/}$mice were crossed to obtain parental genotypes. At the age of $6 \mathrm{wk}$, the diet was shifted from chow to a high fat $\operatorname{diet}(0.15 \%$ cholesterol, $21 \%$ fat, and $0.5 \%$ sodium cholate) for various lengths of time $(3,6,12 \mathrm{wk})$. Plasma was collected by cardiac puncture and stored at $-80{ }^{\circ} \mathrm{C}$ until assayed. Aortas were harvested from perfused or non-perfused animals, depending on the subsequent immunohistochemical method required.

\section{Immunofluorescence}

For immunofluorescence, aortic root cross sections were prepared from 4\% PFA in $0.9 \%$ salineperfused mice sacrificed with ether anaesthesia. Serial $6-\mu \mathrm{m}$ thick cryostat sections were prepared. Aortic root cryosections were blocked with $10 \%$ goat serum and incubated with primary antibodies against $\alpha 4 \beta 7$ integrin (1:500) (clone DATK32; Biolegend, CA, USA), VCAM-1 (1:500) (sc-1504, Santa Cruz, CA, USA) and MAdCAM-1 (1:100) (clone MECA-367; AbD Serotec, Kidlington, UK) or negative IgG control for $16 \mathrm{~h}$ at 4 ${ }^{\circ} \mathrm{C}$. Immunoreactivity was visualized using Alexa Fluor 488-conjugated or/and Alexa Fluor 594-conjugated secondary antibodies (Invitrogen, OR, USA) at a final dilution of 1:1000. For double immunofluorescence, slides were incubated with a rabbit anti-mouse CD31 antibody (1:100) (ab28364, Abcam, Cambridge, UK). This was followed by incubation with FITC-conjugated secondary antibodies. The slides were counterstained with DAPI (5 mg/mL, Sigma) and mounted in glycerin jelly medium for microscopy (Eclipse Ti, Nikon).

Aortic digestion and flow cytometry

Harvested aortas were enzymatically digested with $125 \mathrm{U} / \mathrm{mL}$ collagenase type XI, $60 \mathrm{U} / \mathrm{mL}$ hyaluronidase type I-s, $60 \mathrm{U} / \mathrm{mL}$ DNAse1, and $450 \mathrm{U} / \mathrm{mL}$ collagenase type I (all enzymes, Sigma) in PBS containing $20 \mathrm{mmol} / \mathrm{L} \mathrm{HEPES}$ for 30 minutes at $37^{\circ} \mathrm{C}$ [5]. After digestion, cells were filtered using a $70-\mu \mathrm{m}$ strainer cell strainer (BD Biosciences). The aortic macrophages and $\mathrm{T}$ cells were isolated from single cell suspensions of aorta with FACS sorting (BD FACSAria). Sorted cells were staining with $\alpha 4 \beta 7$ integrin (clone DATK32; Biolegend) for flow cytometric analysis.

Immunohistochemistry

For immunohistochemistry, the aortas were snap-frozen in OCT (Sakura Finetek). Six micrometre thick cryostat sections were cut in series and transferred to slides (SuperFrost Plus; Menzel-Glaser, Germany), airdried and fixed in $10 \%$ acetone for $10 \mathrm{~min}$ at room temperature. After blocking the endogenous peroxidase activity by incubation with $3 \%$ hydrogen peroxidase and washing in PBST ( $1 \times$ PBS with $0.5 \%$ Tween 20 , pH 7.4), sections were incubated with antibodies against $\alpha 4 \beta 7$ integrin (1:500), VCAM-1 (1:500) and MAdCAM-1 (1:100) for $2 \mathrm{~h}$ at room temperature. Sections were rinsed in PSBT and incubated for 30 min with biotinylated secondary antibodies in a 1:500 dilution. Immunohistochemical staining was developed with 2\% 3,3'-diaminobenzidine (DAB, Sigma-Aldrich Corp) and sections were counterstained with hematoxylin. 


\section{Cellular Physiology and Biochemistry}

Cell Physiol Biochem 2014;33:1876-1887

DOI: $10.1159 / 000362965$

Publisned onine: June Z3, 2014

C) 2014 S. Karger AG, Basel

www.karger.com/cpb

Cell isolation and flow cytometry

PBL were isolated by Ficoll-Paque PLUS (GE Healthcare, Buckinghamshire, UK) density gradient centrifugation and subjected to short washing steps to remove platelets. PBL were used immediately or cultured for 2-3 days in Iscove's Modified Dulbecco's Medium (IMDM) with no serum or cholesterol supplement. Peritoneal macrophages were harvested from mice 3 days after intraperitoneal injection of thioglycollate ( $2 \mathrm{ml}, 4 \%$ ) and plated in Dulbecco's Modified Eagle Medium (DMEM) containing 10\% FBS (Gibco, Grand Island, NY, USA). Floating cells were removed after 4 hours incubation at $37^{\circ} \mathrm{C}$ and adherent cells were used in experiments. For flow cytometry, PBL were obtained as described above and 1-5 $\times 10^{6}$ cells were stained with primary antibodies against $\alpha 4 \beta 7$ integrin (1:100, clone DATK32; BD Horizon, San Diego, CA) in HBSS $/ 0.2 \%$ sodium azide/ $2 \%$ calf serum for $20-30$ min at $4 \stackrel{\circ}{\circ}$. After washing, the stained cells were analysed with a FACSCalibur flow cytometer (BD Biosciences, San Jose, CA) and analysed with FlowJo software.

\section{Atherosclerotic lesion analysis}

Aortas or aortic root cross sections were fixed overnight with $4 \%$ paraformaldehyde, washed 3 times with ice-cold PBS, and stained with Oil red-O (Santa Cruz) for 3 hours. The aortic plaque area was quantified manually using Analysis FIVE software by an investigator blinded to the genotypes.

\section{Plasma lipid analysis}

For plasma lipid analysis, mice were fasted for $16 \mathrm{~h}$ before collecting blood. Plasma was separated by centrifugation and stored at $-80{ }^{\circ} \mathrm{C}$ until further analysis. Plasma concentrations of total cholesterol were determined by a colorimetric assay using Infinity ${ }^{\mathrm{TM}}$ Cholesterol Reagent (Thermo Electron Corp, Waltham, MA).

\section{Determination of native LDL uptake and phagocytosis assays}

To measure lipoprotein uptake, peritoneal macrophages were suspended in DMEM with 10\% FBS and seeded onto sterile coverslips at $1 \times 10^{6}$ cells $/ \mathrm{mL}$. After overnight culture, adherent macrophages were washed with DMEM then incubated for $3 \mathrm{~h}$ with $10 \mu \mathrm{g} / \mathrm{mL}$ Dil-labelled-acetylated LDL (Dil-AcLDL) or Dil-LDL (Molecular Probes) in DMEM containing 2.5\% lipoprotein-deficient serum. Lipoprotein uptake in macrophages was quantified by observation under fluorescence microscopy.

Macrophage-mediated phagocytosis of apoptotic thymocytes was examined by harvesting peritoneal macrophages activated with an intraperitoneal injection of $1 \mathrm{~mL} \mathrm{3 \%}$ aged Brewer's thioglycolate 3 days before collection and plating in DMEM containing 10\% FBS to form a macrophage monolayer. Mouse thymocytes were isolated from the thymus of 6- to 8-wk-old Swiss-Webster mice, incubated with $1 \mathrm{mM}$ dexamethasone in RPMI/10\% FCS for $14 \mathrm{~h}$ to induce apoptosis and labelled with CellTracker (CellTracker ${ }^{\mathrm{TM}}$ Red CMTPX, Molecular Probes). Two million dexamethasone-treated and CellTracker-labelled thymocytes were incubated with macrophage monolayers for $80 \mathrm{~min}$ at $37^{\circ} \mathrm{C}$. Uninternalized thymocytes were removed by washing wells 5 times with ice-cold PBS followed by a $20 \mathrm{~min} 0.25 \%$ trypsin $/ 0.02 \%$ EDTA treatment at $22{ }^{\circ} \mathrm{C}$. Cells were then fixed with $4 \%$ paraformaldehyde in PBS and the presence of internalized apoptotic thymocytes in the macrophage preparation was evaluated by microscopic observation.

\section{Western blotting}

We assayed the phosphorylation status of FAK and MAPK in the peritoneal macrophages of $\beta 7^{+/+} \mathrm{ApoE} / \mathrm{E}^{-/}$ and $37^{-/-} \mathrm{ApoE}^{-/-}$mice fed a HFD for 12 weeks. Samples were obtained by cell lysis of peritoneal macrophages in Nonidet P-40 lysis buffer supplemented with protease inhibitor cocktail (Pierce, Rockford, IL, USA), sodium vanadate (Sigma), and PMSF (Sigma). The Bradford assay was used to determine protein concentration. Protein extracts were separated by $9 \%$ SDS-PAGE and transferred to nitrocellulose membranes. Membranes were blocked with 5\% dry milk in TBS/Tween-20 and incubated with anti-phospho-FAK (Tyr397) (1:1000, \#3283), anti-FAK (1:100, \#3285), anti-phospho-ERK (Thr202/Tyr204) (1: 2000, \#4377), and anti-ERK (1: 1000, \#4695) antibodies, all supplied by Cell Signaling Technology (Danvers, MA, USA). Next, membranes were washed then incubated with goat anti-rabbit IgG-HRP (1: 2000) (Santa Cruz) secondary antibody for $1 \mathrm{~h}$ at room temperature. Blots were developed with SuperSignal West Pico Chemiluminescent Substrate kit (Thermoscientific, USA) and exposed to Fuji Medical X-Ray films (FujiFilm Corp., Japan). ImageJ was used to image analysis (U.S. National Institutes of Health, USA). 


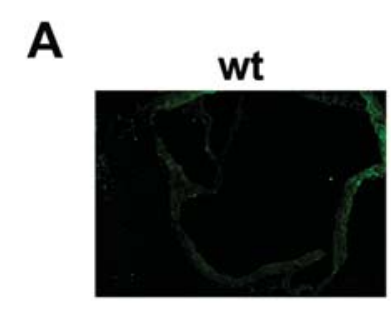

C

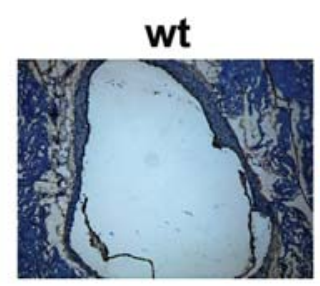

D

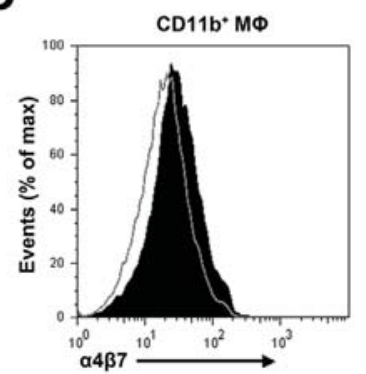

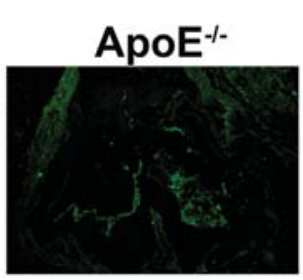

ApoE-1-

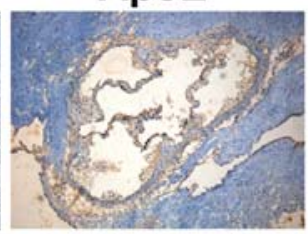

E

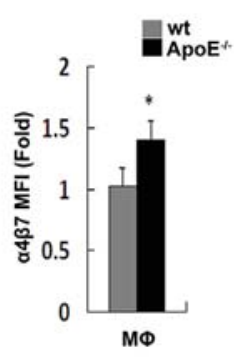

B
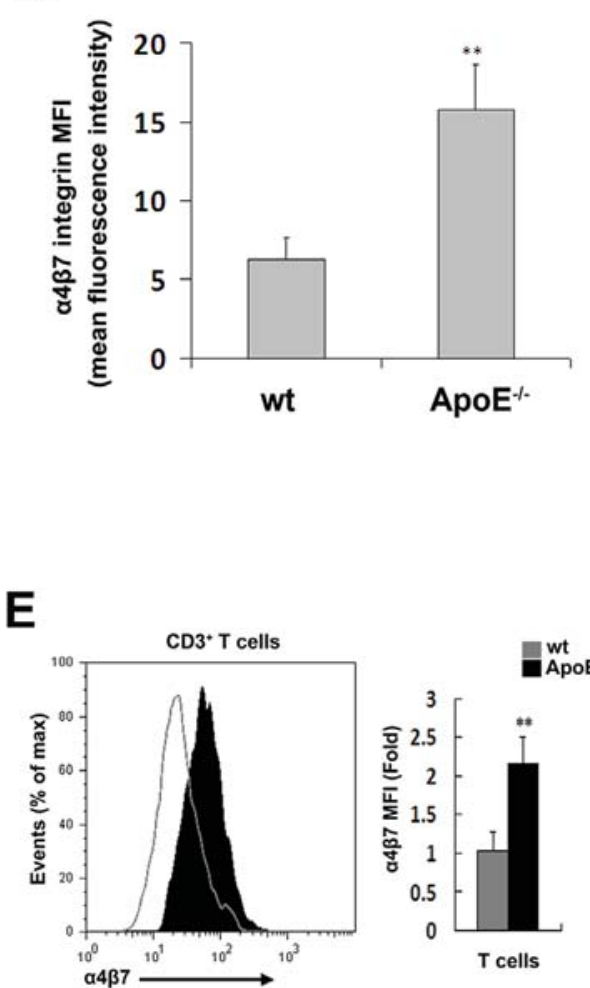

Fig. 1. $\alpha 4 \beta 7$ depositions at atherosclerosis-prone sites in $A p o E^{-/-}$and $\mathrm{C} 57 \mathrm{BL} / 6$ (wt) mice fed a high fat diet for 12 weeks. (A) Representative aortic root cross-sections stained with anti- $\alpha 4 \beta 7$ integrin antibody (green) and nuclear stain DAPI (blue) (Magnification, $\times 100$ ). (B) Comparison of mean fluorescence intensity of C57BL/ 6 (wt) and $\mathrm{ApoE}^{-/}$aortic root cross-sections stained with anti- $\alpha 4 \beta 7$ integrin antibody by immunofluorescence. ${ }^{* *} P<0.01$. (C) Representative immunohistochemistry of aortic root cross-sections stained with anti- $\alpha 4 \beta 7$ integrin antibody (Magnification, $\times 50$ ). (D) Representative flow plot (left) and quantification (right) of $\alpha 4 \beta 7$ integrin expression on aortic CD11 $\mathrm{b}^{+}$macrophages from C57BL/6 (wt) (gray line) and ApoE ${ }^{-/-}$(black filled line) measured by flow cytometry. Quantification of data expressed as fold change in $\alpha 4 \beta 7$ integrin mean fluorescent intensity over C57BL/6 (wt) CD11 b macrophages set as $1 ;{ }^{*} P<0.05$. (E) Representative flow plot (left) and quantification (right) of $\alpha 4 \beta 7$ integrin expression on aortic $\mathrm{CD}^{+} \mathrm{T}$ cells from $\mathrm{C} 57 \mathrm{BL} / 6$ (wt) (gray line) and $\mathrm{ApoE}^{-/-}$(black filled line) measured by flow cytometry. Quantification of data expressed as fold change in $\alpha 4 \beta 7$ integrin mean fluorescent intensity over C57BL/6 (wt) $\mathrm{CD}^{+} \mathrm{T}$ cells set as $1 ;{ }^{* *} \mathrm{P}<0.01$.

\section{Statistical Analysis}

Results are presented as the mean \pm SD from a minimum of 3 replicates. The difference between groups was evaluated by SPSS 17.0 statistical software with Student's t-test when comparing only two groups or assessed by one-way analysis of variance when more than two groups were compared. $P<0.05$ was considered to indicate a statistically significant result.

\section{Results}

$\alpha 4 \beta 7$ integrin is highly expressed in atherosclerosis plaques in ApoE ${ }^{-/-}$mice

We assayed the expression of $\alpha 4 \beta 7$ in atherosclerosis plaques in aortas of HFD fed $\mathrm{ApoE}^{-/}$mice, a widely used model of atherosclerosis in which plasma lipoproteins are elevated due to absence of the lipoprotein-clearing protein ApoE [27]. We fed ApoE ${ }^{-/-}$and 


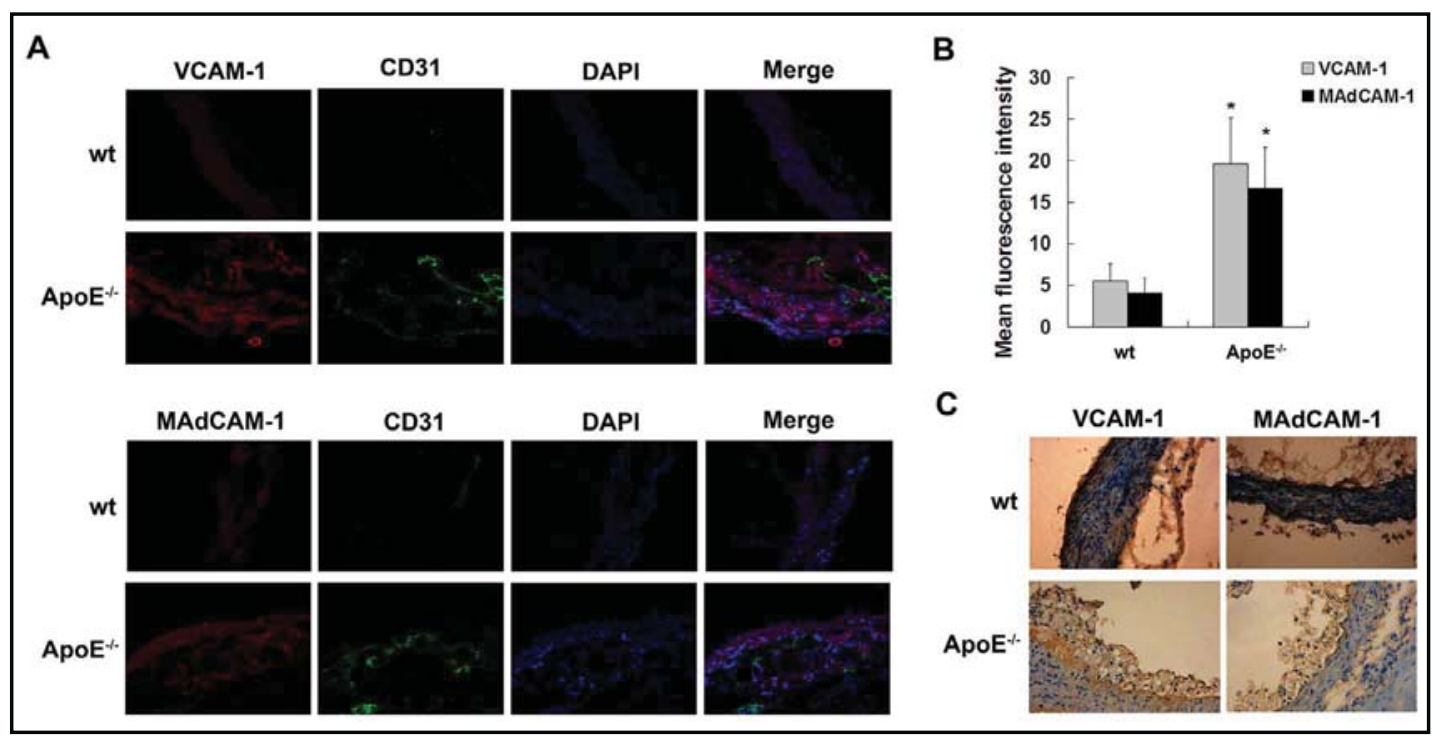

Fig. 2. MAdCAM-1 and VCAM-1 expression in plaques from $A \mathrm{poE}^{-/-}$and $\mathrm{C} 57 \mathrm{BL} / 6$ (wt) mice fed a high fat diet for 12 weeks. (A) Representative aortic root cross-sections double-stained with anti-MAdCAM-1 or anti-VCAM-1 antibody (red) and CD31 (green) and nuclear stain DAPI (blue) (Magnification, ×200). (B) Comparison of mean fluorescence intensity of immunofluorescent anti-VCAM-1 or anti-MAdCAM-1 antibody stained ApoE $\%$ and $\mathrm{C} 57 \mathrm{BL} / 6$ (wt) aortic root cross-sections. ${ }^{*} P<0.05$. (C) Representative immunohistochemistry of aortic root cross-sections stained with anti-VCAM-1 or anti-MAdCAM-1 antibody (Magnification, $\times 200$ ).

control C57BL/6 mice a HFD diet for 12wk, after which we found a strong expression of $\alpha 4 \beta 7$ integrin in atherosclerosis plaques in $\mathrm{ApoE}^{-/}$aortas using immunofluorescent (Fig. 1A) and immunohistochemical detection techniques (Fig. 1C). A quantification of immunofluorescent density confirmed that the expression of $\alpha 4 \beta 7$ was significantly higher in $\mathrm{ApoE}^{-/-}$than in control C57BL/6 aortic cross sections (Fig. 1B). Based on the strong association of macrophages and T cells with atherosclerosis, we examined $\alpha 4 \beta 7$ expression on macrophages and total T cells in aortas of C57BL/6 and $\mathrm{ApoE}^{-/-}$mice after a HFD diet for $12 \mathrm{wk}$. We found increased $\alpha 4 \beta 7$ integrin expression in $\mathrm{CD}_{11 \mathrm{~b}^{+} \text {macrophages and } \mathrm{CD}^{+} \mathrm{T}}$ cells by flow cytometry (Fig. 1D and E).

VCAM-1 and MAdCAM-1 are upregulated in atherosclerosis plaques in ApoE-/ mice

We next examined the expression of $\alpha 4 \beta 7$ ligands MAdCAM- 1 and VCAM- 1 in atherosclerosis plaques from $12 \mathrm{wk}$ HFD fed ApoE $\mathrm{E}^{--}$and C57BL/6 mice. Compared to control aortas, MAdCAM-1 and VCAM-1 expression appeared higher in $\mathrm{ApoE}^{-/-}$mice using immunofluorescent (Fig. 2A) and immunohistochemical detection techniques (Fig. 2C). A quantification of fluorescence units confirmed that MAdCAM- 1 and VCAM-1 expression in ApoE $/$ a aortic cross sections was higher than in control C57BL/6 aortas (Fig. 2B). Furthermore, as shown in Figure 2A, MAdCAM-1 and VCAM-1 staining colocalized in endothelial cells within plaques, suggesting that alterations in MAdCAM-1 and VCAM-1 expression in plaque lesions are mainly caused by endothelial cells.

Upregulation of $\alpha 4 \beta 7$ integrin is associated with atherogenic lesion progression

To establish whether $\alpha 4 \beta 7$ integrin expression in diet-induced $\mathrm{ApoE}^{-/}$plaques was related to the pathogenesis of atherosclerosis, we determined the temporal expression profile of $\alpha 4 \beta 7$ integrin in PBL of ApoE ${ }^{-/}$mice fed a HFD for 12 wks. Compared to control C57BL/ 6 lymphocytes, $\alpha 4 \beta 7$ expression in lymphocytes from HFD fed $\mathrm{ApoE}^{-/-}$animals was higher at all time points measured, namely at 3,6 and 12 weeks (Fig. 3A). Furthermore, $\alpha 4 \beta 7$ expression in ApoE $\%$ PBL increased steadily over the $12 \mathrm{wk}$ observational period (Fig. 3B). 
Fig. 3. Upregulation of $\alpha 4 \beta 7$ is associated with atherosclerosis progression. $\mathrm{ApoE}^{-/}$and $\mathrm{C} 57 \mathrm{BL} / 6$ (wt) mice were fed a high fat diet, and analysed at 3 , 6 and 12 weeks. (A) Representative FACS analysis of anti- $\alpha 4 \beta 7$ integrin stained $\mathrm{ApoE}^{-/}$and $\mathrm{C} 57 \mathrm{BL} / 6$ (wt) PBL. Isotype control staining (Iso) is included throughout. (B) Median fluorescence intensity of $\alpha 4 \beta 7$ expression on PBL from C57BL/6 (wt) and ApoE $E^{-/}$mice. The data are expressed as mean \pm SD ( $\mathrm{n}=5$ separate mice). The data are expressed as means \pm SD ( $=5$ separate mice) ${ }^{*} P<0.05,{ }^{* *} P<0.01$. (C) Representative flow plot of $\alpha 4 \beta 7$ integrin expression on aortic $\mathrm{CD}_{14} 4^{+}$monocytes and $\mathrm{CD}^{+} \mathrm{T}$ cells from C57BL/6 (wt) (gray line) and $\mathrm{ApoE}^{-/-}$(black line) after 12 weeks of HFD measured by flow cytometry. (D) Representative Oil Red 0 staining of aortic root cross-sections from $\mathrm{ApoE}^{-/}$ and C57BL/6 (wt) mice fed a high fat diet for 12 weeks (Magnification, $\times 50$ ). (E)

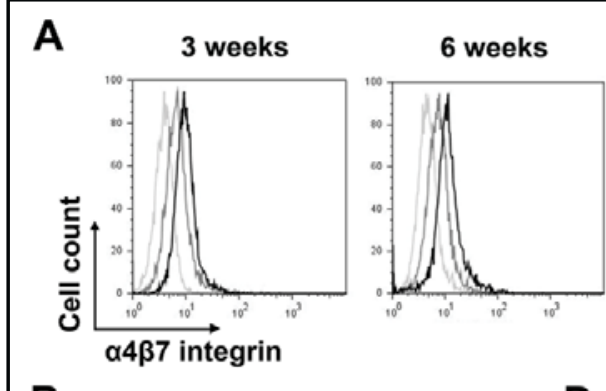

B

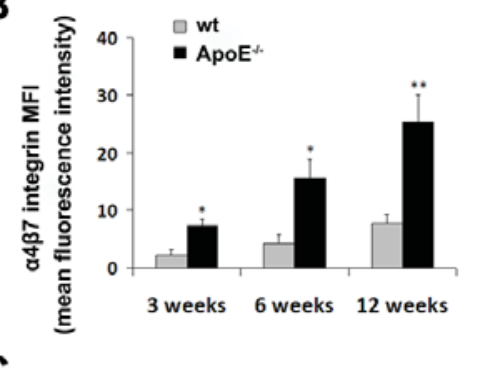

D

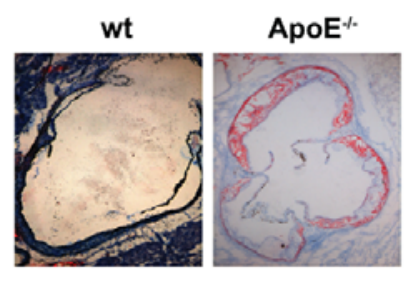

C

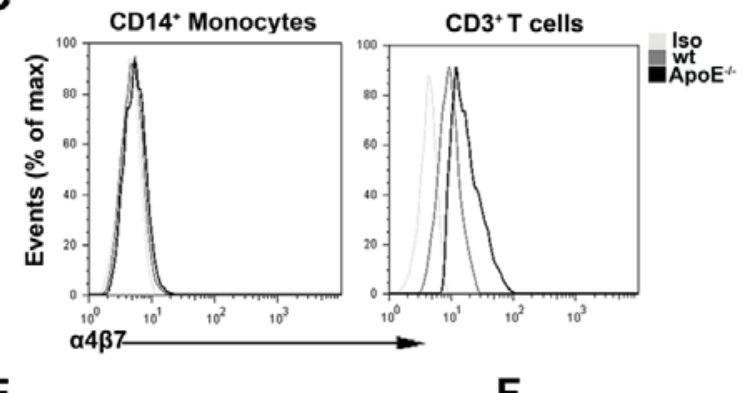

$E$

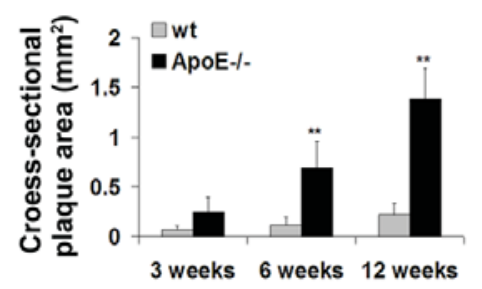

F

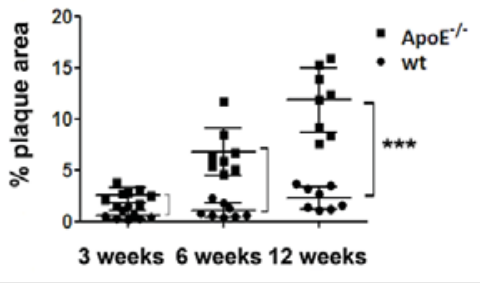

Comparison of plaque area in $\mathrm{ApoE}^{-/-}$and C57BL/6 (wt) aortic root cross-sections. ${ }^{* *} P<0.01$. (F) Ratio of lesion area to median aortic area in $\mathrm{ApoE}^{-/}$and $\mathrm{C} 57 \mathrm{BL} / 6$ aortas. Individual values are given for each time point and long horizontal bars designate the median values and short horizontal bars designate the SD values. ${ }^{* * *} P<0.001$.

In addition, we observed $\alpha 4 \beta 7$ integrin was highly expressed in T cells but not in monocytes (Fig. 3C). An analysis of Oil Red 0 stained cross sections (Fig. 3D) revealed a steadily increasing mean cross-sectional (Fig. 3E) and relative (Fig. 3F) plaque area in $A p o E^{-/-}$aortas. The mean $\mathrm{ApoE}^{-/-}$cross-sectional plaque area was significantly higher than that of $\mathrm{C} 57 \mathrm{BL} / 6$ aortas at 6 and 12 wk (Fig. 3E) and the mean $\mathrm{ApoE}^{-/-}$relative plaque area was significantly higher than the control goup at 3, 6 and $12 \mathrm{wk}$ (Fig. 3F). In summary, the increase in lymphocyte $\alpha 4 \beta 7$ integrin expression occurs in parallel with an increase in plaque area in HFD fed $\mathrm{ApoE}^{-/-}$aortas and raises the possibility that elevated $\alpha 4 \beta 7$ integrin expression in lymphocytes promotes the formation of atherosclerotic lesions.

Deletion of $\alpha 4 \beta 7$ integrin in mice inhibits the formation of atherosclerotic lesions

To further explore the potential pro-atherogenic role of $\alpha 4 \beta 7$ integrin during atherosclerotic lesion progression, we removed $\alpha 4 \beta 7$ integrin by genetic deletion of 
A

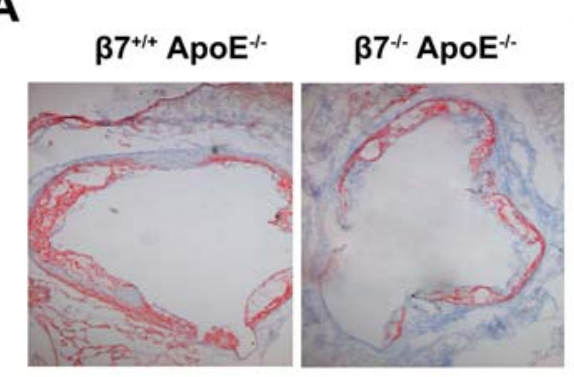

C

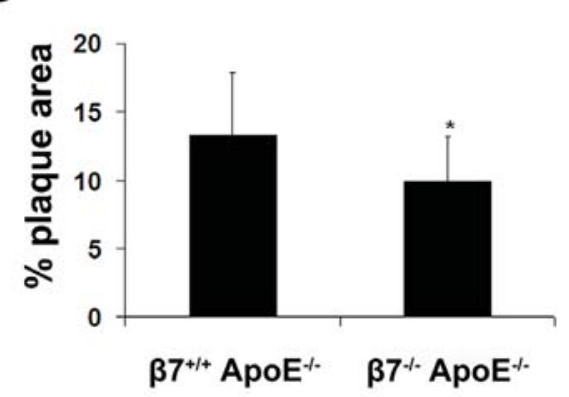

B

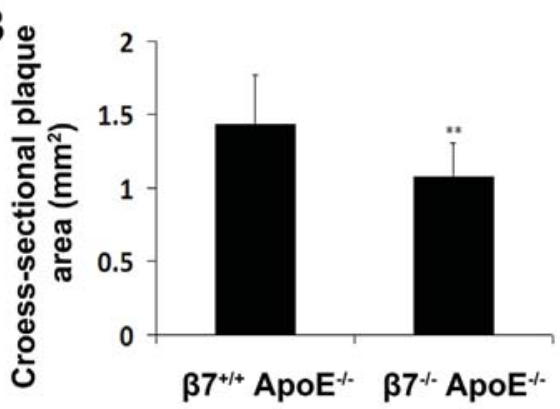

D

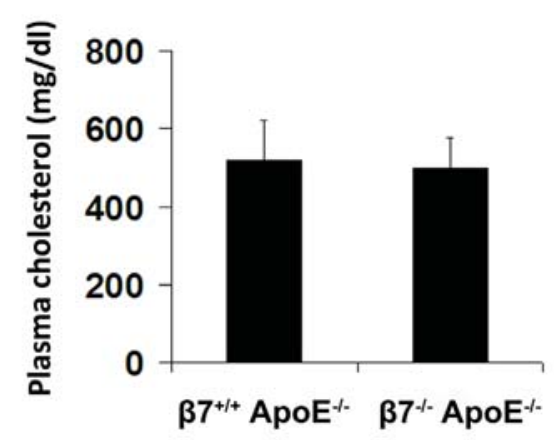

Fig. 4. Deletion of $\alpha 4 \beta 7$ in mice inhibits the formation of atherosclerotic lesions. $\beta 7^{+/+} A p o E^{-/-}$and $\beta 7^{-/ A p o E^{-/}}$mice were sacrificed after being fed a high fat diet for 12 weeks. (A) Representative Oil Red 0 staining of $\beta 7^{+/+} \mathrm{ApoE}^{-/}$and $\beta 7^{\%} \mathrm{ApoE} \mathrm{E}^{-/}$aortic root cross-sections (Magnification, $\times 50$ ). (B) Cross-sectional plaque area in $\beta 7^{+/+} \mathrm{ApoE} \mathrm{E}^{-/}$and $\beta 7^{-/} \mathrm{ApoE^{-/ }}$ aortic roots. The data are expressed as mean $\pm \mathrm{SD}$. ${ }^{* *} P<0.01$. (C) Ratios of lesion area over media area of $\beta 7^{+/+} \mathrm{ApoE} \mathrm{E}^{-/-}$and $\beta 7^{-/-} \mathrm{ApoE}^{-/}$aortas. ${ }^{*} P<0.05$. (D) Plasma cholesterol levels in $\beta 7^{+/+} \mathrm{ApoE}{ }^{-/}$and $\beta 7^{-/} \mathrm{ApoE} \mathrm{E}^{-/}$mice. The data are expressed as mean $\pm \mathrm{SD}(\mathrm{n}=5$ separate mice $)$.

the $\beta 7$ chain in the ApoE $/-$ mouse. We found that a deletion of $\beta 7$ integrin reduced the size of HFD induced atherosclerotic lesions in $\mathrm{ApoE}^{-/}$mice (Fig. 4A). At $12 \mathrm{wk}$, both the mean cross-sectional and relative plaque areas were significantly lower in $\beta 7^{-/} \mathrm{ApoE}^{-/}$double mutants than those of $\beta 7^{+/+} \mathrm{ApoE}^{-/-}$mice (Fig. $4 \mathrm{~B}$ and $\mathrm{C}$ ). To exclude the possibility of an alteration in lipid metabolism caused by the $\beta 7$ integrin deletion, plasma cholesterol levels in HFD fed ApoE $\mathrm{E}^{-/}$and $\beta 7^{-\%} \mathrm{ApoE}^{-/}$mice were compared. There was no significant difference in cholesterol levels between the two genotypes (Fig. 4D). This has also been observed in HFD fed ApoE ${ }^{-/}$mice lacking $\alpha 2 \beta 1$ integrin [10] or integrin ligand ICAM-1 [9]. Together these results strongly suggest a pro-atherogenic function for $\alpha 4 \beta 7$ integrin in the formation of atherosclerotic lesions.

$\alpha 4 \beta 7$ integrin deficiency inhibits phagocytic activity of peritoneal macrophages

Integrins are potentially involved in all phases of atherogenesis [1]. A key processes in early atherosclerosis is the formation of foam cells when monocyte-derived phagocytes located in atherosclerotic lesions ingest and process apoB-LPs [4]. To examine whether $\alpha 4 \beta 7$ integrin modulates the uptake of native or modified lipoproteins, we incubated peritoneal macrophages from HFD fed ApoE ${ }^{-/}$and $37^{-/} \mathrm{ApoE}^{-/}$mice in the presence of Dil-AcLDL or Dil-LDL and quantified the amount of internalized ligand associated using fluorescent image analysis. We found that $\beta 7^{-/} \mathrm{ApoE}^{-/}$macrophages displayed a reduced acetylated LDL and native LDL uptake (Fig. 5A and B). These results suggest that the reduced atherosclerotic plaque size upon $\alpha 4 \beta 7$ integrin deletion could be caused by a reduced lipoprotein uptake in macrophages. We next investigated whether phagocytic function of macrophages is affected by $\beta 7$ integrin loss. In advanced atherosclerosis, the reduced uptake of apoptotic cells by macrophages is thought to contribute to the overall inflammation at the atherosclerotic 
Fig. 5. $\alpha 4 \beta 7$ deficiency inhibits phagocytic activity of peritoneal macrophages. (A) Dil-AcLDL and Dil-LDL uptake was measured in $\beta 7^{+/+} \mathrm{ApoE}^{-/-}$and $\beta 7^{-/-} \mathrm{ApoE}^{-/-}$ peritoneal macrophages. (B) Dil-AcLDL and Dil-LDL fluorescence expressed as arbitrary units (AU) per macrophage. ${ }^{*} P<0.05$. (C) $\beta 7^{+/+} \mathrm{ApoE}^{-/-}$and $\beta 7^{-/-} \mathrm{ApoE}^{-/-}$ peritoneal macrophage phagocytosis of CellTracker red labelled apoptotic thymocytes. (D) The numbers of CellTracker red-positive cells per $\beta 7^{+/+} \mathrm{ApoE}^{-/}$or $\beta 7^{-/} \mathrm{ApoE}^{-/}$peritoneal macrophage. The data are expressed as mean \pm SD $(n=5$ separate mice). ${ }^{*} P<0.05$.

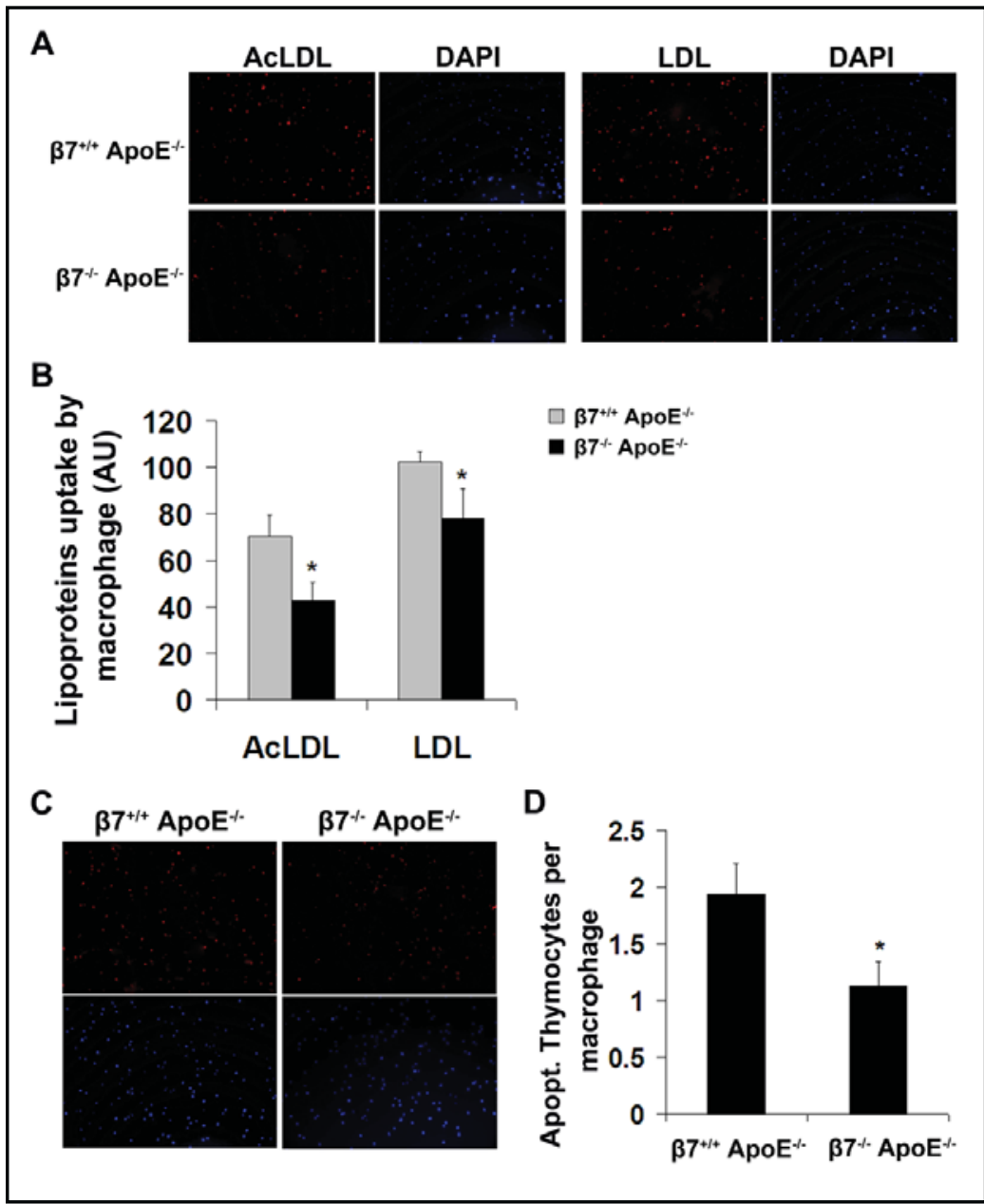

plaque as a result of the release of pro-inflammatory molecules from dead cells [28]. We incubated $\mathrm{ApoE}^{\%}$ or $\beta 7^{\%} \mathrm{ApoE}^{\%}$ macrophages with apoptotic thymocytes labelled with CellTracker red and determined by fluorescent microscopy the number of apoptotic thymocytes internalized in the macrophages. The number of CellTracker red positive apoptotic cells internalized per macrophage was significantly lower in macrophages lacking $\beta 7$ integrin (Fig. 5D). Together these results demonstrate an overall reduction of phagocytic avidity in $\beta 7^{-} / \mathrm{ApoE}^{-/}$macrophages.

\section{Reduced phosphorylated FAK and ERK1/2 in $\alpha 4 \beta 7$ deficient macrophages.}

To explore the signalling pathway changes leading to the atheroprotective phenotype observed in $37^{-/}$ApoE ${ }^{-/}$mice fed a 12 week HFD, we assessed the changes in phosphorylation levels of peritoneal macrophage ERK1/2 and FAK, two downstream signalling effectors of integrin engagement. Indeed, we found reduced levels of phosphorylated FAK (Fig. 6A) and ERK1/2 (Fig. 6B) in $\beta 7^{-/} \mathrm{ApoE}^{-/}$macrophages indicating a reduced activation of these two pathways, possibly reflecting the lack of engagement of $\alpha 4 \beta 7$ integrin in these cells due to the absence of $\beta 7$ integrin .

\section{Discussion}

Efficient lymphocyte homing to normal tissues and sites of inflammation is an essential part of innate immunity. Homing and recruitment are driven by multistep pathways using selective expression of chemokines, pro-inflammatory cytokines and 
Fig. 6. FAK and MAPK/ERK activity is inhibited in peritoneal macrophages of $\alpha 4 \beta 7$-deficient mice. (A) ERK1/2 phosphorylation in $\beta 7^{+/+} \mathrm{ApoE}^{-/}$or $\beta 7^{-/}$ApoE ${ }^{-/}$peritoneal macrophages displayed by Western blotting and quantified by densitometry. ${ }^{* *} P<0.01$. (B) FAK phosphorylation in $\beta 7^{+/+} \mathrm{ApoE}^{-/-}$or $\beta 7^{-} \mathrm{ApoE}^{-/}$peritoneal macrophages displayed by Western blotting and quantified by densitometry. ${ }^{* *} P<0.01$.

mediators and various adhesion proteins and molecules. Importantly from a therapeutic standpoint, these pathways are also active in diseases characterized by inflammatory cell infiltration. Therefore, a better understanding of pathways that mediate lymphocyte homing could lead to the discovery of novel therapeutic targets for immunoinflammatory diseases such as atherosclerosis.

In addition to its role in homing lymphocytes to pancreatic and gutassociated lymphoid tissues $[22,29,30]$ the $\alpha 4 \beta 7$ integrin-MAdCAM- 1 adhesion pathway has also been implicated in activated lymphocyte recruitment to lymphoid [22-24] as well as extra

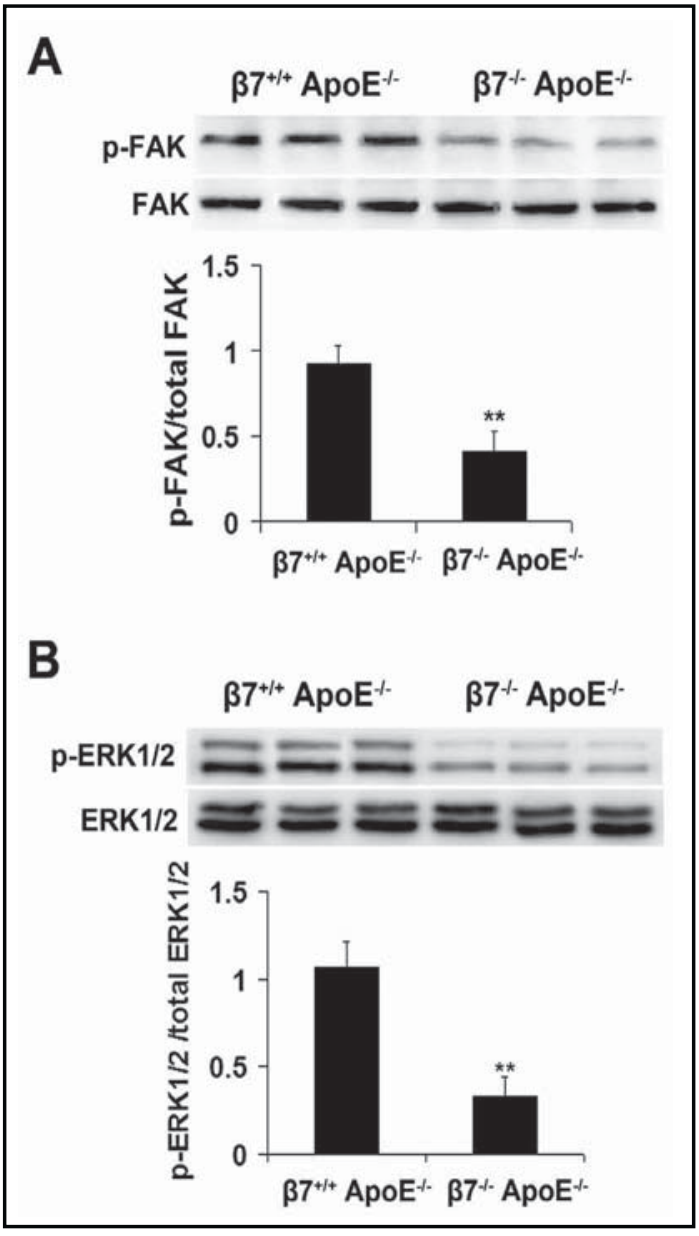

lymphoid sites of inflammation [31]. Our study reveals a possible involvement of the $\alpha 4 \beta 7$ integrin-MAdCAM-1 adhesion pathway in yet another extra lymphoid site of inflammation, namely arterial lesions. We observed an increased $\alpha 4 \beta 7$ integrin, MAdCAM- 1 and VCAM-1 expression in atherosclerotic plaques of HFD fed ApoE $/ /$ mice, a widely used model of atherosclerosis. Furthermore, PBL $\alpha 4 \beta 7$ integrin expression increased concurrently with aortic plaque size, and therefore atherosclerosis progression. An increased $\alpha 4 \beta 7$ integrin expression in PBL along with high MAdCAM-1 levels in murine endothelial cells exposed to pro-inflammatory cytokines [25], would promote plaque formation at inflammatory sites. When we genetically deleted $\beta 7$ integrin from $\mathrm{ApoE}^{-/-}$mice, plaque size in HFD animals decreased significantly, further supporting a pro-atherogenic role for $\alpha 4 \beta 7$ integrin. These observations are compatible with the antibody mediated inhibition of $\alpha 4$ integrin [32], which resulted in a reduction in monocyte recruitment to atherosclerotic plaques in $\mathrm{ApoE}^{-/}$mice. We speculate this was due to a reduced availability of pro-atherogenic $\alpha 4 \beta 7$ integrin complexes.

We found that $\beta 7^{-/ A p o E^{-/}}$macrophages displayed a reduced acetylated $\mathrm{LDL}$ and native LDL uptake, indicating that reduced plaque formation in HFD $\beta 7^{\%} \mathrm{ApoE}^{-/}$mice could reflect a reduced ability of macrophages to accumulate lipoproteins and form foam cells during the critical initiation steps of atherosclerosis. However, we also found reduced phagocytic activity in $\beta 7^{-/} \mathrm{ApoE}^{-/-}$macrophages, meaning that at later stages $\alpha 4 \beta 7$ integrin could be involved in the elimination of dying cells, thereby limiting lesion expansion and atherosclerosis progression. This last result contrasts with the pro-atherogenic role of $\alpha 4 \beta 7$ revealed by our study of the $\beta 7 \%$ po: ${ }^{-}$double deficient mouse. However, it also points to the limitations of the mouse model for studies of late stages of atherosclerosis [4]. It is nevertheless conceivable that in a dynamic disease such as atherosclerosis, a single molecule 


\section{Cellular Physiology and Biochemistry}

Cell Physiol Biochem 2014;33:1876-1887

\begin{tabular}{l|l}
\hline DOI: $10.1159 / 000362965$ & C 2014 S. Karger AG, Basel
\end{tabular}

www.karger.com/cpb

Zhi et al.: $\alpha 4 \beta 7$ Integrin Involved in Atherosclerosis

could have a pro-atherogenic role in the early lesion stage followed by an atheroprotective role in the fibrous plaque stage.

Integrins modulate numerous signalling cascades affecting key biological processes. $\alpha 4 \beta 7$ integrin induces primary lung fibroblast differentiation by activating FAK and MAPKassociated signalling pathways upon binding to cellular fibronectin [33]. Along similar lines, we observed a reduced activation status of cytoplasmic signalling molecules FAK and ERK1/2 in $37^{-/ A p o E^{-/}}$peritoneal macrophages. More elaborate experiments would be required to confirm that the reduced FAK and ERK1/2 phosphorylation observed here is a direct consequence of $\beta 7$ deficiency.

In conclusion, this study implicates the involvement of $\alpha 4 \beta 7$ integrin in a number of steps during atherogenesis in mouse. A further characterization of its pro-atherogenic roles could identify potential therapies for atherosclerosis.

\section{Acknowledgements}

This work was supported by the Program of Science and Technology Commission of Shanghai Municipality 13ZR1414500 and 11ZR1433200; the Program of Putuo District Science and Technology Commission of Shanghai, No. B121.

\section{References}

1 Blankenberg S, Barbaux S, Tiret L: Adhesion molecules and atherosclerosis. Atherosclerosis 2003;170:191203.

-2 Johnson JL, Newby AC: Macrophage heterogeneity in atherosclerotic plaques. Current opinion in lipidology 2009;20:370-378.

-3 Paulson KE, Zhu SN, Chen M, Nurmohamed S, Jongstra-Bilen J, Cybulsky MI: Resident intimal dendritic cells accumulate lipid and contribute to the initiation of atherosclerosis. Circ Res 2010;106:383-390.

- Moore KJ, Tabas I: Macrophages in the pathogenesis of atherosclerosis. Cell 2011;145:341-355.

5 Galkina E, Kadl A, Sanders J, Varughese D, Sarembock IJ, Ley K: Lymphocyte recruitment into the aortic wall before and during development of atherosclerosis is partially L-selectin dependent. J Exp Med 2006;203:1273-1282.

6 Hoshiga M, Alpers CE, Smith LL, Giachelli CM, Schwartz SM: Alpha-v beta-3 integrin expression in normal and atherosclerotic artery. Circ Res 1995;77:1129-1135.

7 Ley K, Huo YQ: VCAM-1 is critical in atherosclerosis. J Clin Invest 2001;107:1209-1210.

8 Nakashima Y, Raines EW, Plump AS, Breslow JL, Ross R: Upregulation of VCAM-1 and ICAM-1 at atherosclerosis-prone sites on the endothelium in the ApoE-deficient mouse. Arterioscler Thromb Vasc Biol 1998;18:842-851.

-9 Collins RG, Velji R, Guevara NV, Hicks MJ, Chan L, Beaudet AL: P-Selectin or intercellular adhesion molecule (ICAM)-1 deficiency substantially protects against atherosclerosis in apolipoprotein E-deficient mice. J Exp Med 2000;191:189-194.

10 Grenache DG, Coleman T, Semenkovich CF, Santoro SA, Zutter MM: Alpha2beta1 integrin and development of atherosclerosis in a mouse model: assessment of risk. Arterioscler Thromb Vasc Biol 2003;23:21042109.

11 Merched A, Tollefson K, Chan L: Beta2 integrins modulate the initiation and progression of atherosclerosis in low-density lipoprotein receptor knockout mice. Cardiovasc Res 2010;85:853-863.

12 Weng S, Zemany L, Standley KN, Novack DV, La Regina M, Bernal-Mizrachi C, Coleman T, Semenkovich CF: Beta3 integrin deficiency promotes atherosclerosis and pulmonary inflammation in high-fat-fed, hyperlipidemic mice. Proc Natl Acad Sci U S A 2003;100:6730-6735.

13 Lefer AM: Role of the beta2-integrins and immunoglobulin superfamily members in myocardial ischemiareperfusion. Ann Thorac Surg 1999;68:1920-1923. 


\section{Cellular Physiology and Biochemistry}

Cell Physiol Biochem 2014;33:1876-1887

\begin{tabular}{l|l}
\hline DOI: $10.1159 / 000362965$ & C 2014 S. Karger AG, Basel
\end{tabular}

Zhi et al.: $\alpha 4 \beta 7$ Integrin Involved in Atherosclerosis

-14 Lopez-Rodriguez C, Chen HM, Tenen DG, Corbi AL: Identification of Sp1-binding sites in the CD11c (p150,95 alpha) and CD11a (LFA-1 alpha) integrin subunit promoters and their involvement in the tissuespecific expression of CD11c. Eur J Immunol 1995;25:3496-3503.

-15 Lynam E, Sklar LA, Taylor AD, Neelamegham S, Edwards BS, Smith CW, Simon SI: Beta2-integrins mediate stable adhesion in collisional interactions between neutrophils and ICAM-1-expressing cells. J Leukoc Biol 1998;64:622-630.

-16 Rose DM, Grabovsky V, Alon R, Ginsberg MH: The affinity of integrin alpha(4)beta(1) governs lymphocyte migration. J Immunol 2001;167:2824-2830.

17 Moiseeva EP: Adhesion receptors of vascular smooth muscle cells and their functions. Cardiovasc Res 2001, 52:372-386.

18 Schwartz EA, Bizios R, Medow MS, Gerritsen ME: Exposure of human vascular endothelial cells to sustained hydrostatic pressure stimulates proliferation. Involvement of the alphaV integrins. Circ Res 1999;84:315-322.

19 Walsh GM, Symon FA, Lazarovils AL, Wardlaw AJ: Integrin alpha 4 beta 7 mediates human eosinophil interaction with MAdCAM-1, VCAM-1 and fibronectin. Immunology 1996;89:112-119.

20 Yang Y, Harrison JE, Print CG, Lehnert K, Sammar M, Lazarovits A, Krissansen GW: Interaction of monocytoid cells with the mucosal addressin MAdCAM-1 via the integrins VLA-4 and LPAM-1. Immunol Cell Biol 1996;74:383-393.

21 Ruegg C, Postigo AA, Sikorski EE, Butcher EC, Pytela R, Erle DJ: Role of integrin alpha 4 beta 7/alpha 4 beta P in lymphocyte adherence to fibronectin and VCAM-1 and in homotypic cell clustering. J Cell Biol 1992;117:179-189.

22 Xu B, Cook RE, Michie SA: Alpha4beta7 integrin/MAdCAM-1 adhesion pathway is crucial for B cell migration into pancreatic lymph nodes in nonobese diabetic mice. J Autoimmun 2010;35:124-129.

-23 Briskin M, WinsorHines D, Shyjan A, Cochran N, Bloom S, Wilson J, McEvoy LM, Butcher EC, Kassam $\mathrm{N}$, Mackay CR et al: Human mucosal addressin cell adhesion molecule-1 is preferentially expressed in intestinal tract and associated lymphoid tissue. Am J Pathol 1997;151:97-110.

24 Hanninen A, Taylor C, Streeter PR, Stark LS, Sarte JM, Shizuru JA, Simell O, Michie SA: Vascular addressins are induced on islet vessels during insulitis in nonobese diabetic mice and are involved in lymphoid cell binding to islet endothelium. J Clin Invest 1993;92:2509-2515.

-25 Sikorski EE, Hallmann R, Berg EL, Butcher EC: The Peyer's patch high endothelial receptor for lymphocytes, the mucosal vascular addressin, is induced on a murine endothelial cell line by tumor necrosis factor-alpha and IL-1. J Immunol 1993;151:5239-5250.

26 Wagner N, Lohler J, Kunkel EJ, Ley K, Leung E, Krissansen G, Rajewsky K, Muller W: Critical role for beta7 integrins in formation of the gut-associated lymphoid tissue. Nature 1996;382:366-370.

-27 Piedrahita JA, Zhang SH, Hagaman JR, Oliver PM, Maeda N: Generation of mice carrying a mutant apolipoprotein E gene inactivated by gene targeting in embryonic stem cells. Proc Natl Acad Sci U S A 1992;89:4471-4475.

28 Fadok VA, Chimini G: The phagocytosis of apoptotic cells. Semin Immunol 2001;13:365-372.

29 Nakache M, Berg EL, Streeter PR, Butcher EC: The mucosal vascular addressin is a tissue-specific endothelial cell adhesion molecule for circulating lymphocytes. Nature 1989;337:179-181.

- 30 Streeter PR, Berg EL, Rouse BT, Bargatze RF, Butcher EC: A tissue-specific endothelial cell molecule involved in lymphocyte homing. Nature 1988;331:41-46.

-31 O'Neill JK, Butter C, Baker D, Gschmeissner SE, Kraal G, Butcher EC, Turk JL: Expression of vascular addressins and ICAM-1 by endothelial cells in the spinal cord during chronic relapsing experimental allergic encephalomyelitis in the Biozzi AB/H mouse. Immunology 1991;72:520-525.

- 32 Patel SS, Thiagarajan R, Willerson JT, Yeh ET: Inhibition of alpha4 integrin and ICAM-1 markedly attenuate macrophage homing to atherosclerotic plaques in ApoE-deficient mice. Circulation 1998;97:75-81.

-33 Kohan M, Muro AF, White ES, Berkman N: EDA-containing cellular fibronectin induces fibroblast differentiation through binding to alpha4beta7 integrin receptor and MAPK/Erk 1/2-dependent signaling. Faseb J 2010;24:4503-4512. 